\title{
Novel Theory of Mathematical Pendulum Part 2
}

\author{
Zdzisław Pluta, Tadeusz Hryniewicz* \\ Koszalin University of Technology, Raclawicka 15-17, 75-620 Koszalin, Poland \\ *E-mail address: Tadeusz.Hryniewicz@tu.koszalin.pl
}

„Master content, no matter the words" Katon the Elder

\begin{abstract}
The paper is concerned on a new adequate theory of a simple mathematical pendulum. Part 1 of the paper was devoted to the behaviour of pendulum in particular points, that is central and terminal/extremum ones. This Part 2 of the theory begins with the analysis of path length of the pendulum weight. Then the kinetics of the pendulum weight is analyzed by separating and the descriptions of differentiated motion of this body in the consecutive neighbouring space-times corresponding with particular quarter-periods. It is about accelerated free variable motion and the following after it a retarded motion of this kind, and then again accelerated, etc. In the summary, further elaborations in the subject are forecasted, regarding both dynamics and energy of the flat mathematical pendulum. It is indicated that the necessity to "rethink" many existent theories is of importance.
\end{abstract}

Keywords: Mathematical pendulum; Inertia; Kinetics; Potential field; Stable state; Unstable state

\section{INTRODUCTION}

The work is devoted to the simple mathematical pendulum with the first part given in [1]. The existent theory of the mathematical pendulum is commonly known and its critics was presented earlier $[2,3]$.

A motivation of the subject was revealed in the first part of the work. Part 2 of the article is to provide with the solution covering the path length and kinetic derivatives of the pendulum weight leaving the energy and dynamics problems for consideration in the following studies.

The shortcomings of the existent theory provide a sufficient basis to undertake the subject studies. A new theory of mathematical pendulum will be presented, without the drawbacks of up-to-date approach to describe the real pendulum behaviours. 


\section{PATH/TRACK LENGTH OF THE PENDULUM WEIGHT}

The path length - that should be noticed - is not the same as the length of track/trajectory of a material body; in reference to the described reality the body is the weight of pendulum. The notion of path length was explained also in the previous work [4], devoted to the kinetics of tool edge fixed flexibly.

That first physical magnitude (path length) is a primary magnitude which then may be brought to other, derivative magnitudes, by the differentiating. Differentiating the track of the material body makes no sense because its derivatives (considered against time) do not correspond with the consecutive kinetic magnitudes. Only differentiating the path length against time results in obtaining the derivatives of kinetic time characteristics. The value of path length should be first zero, then positive and growing (progressively or degressively), because only in that case the magnitude has a physical sense; no negative magnitudes should be assumed. All those mentioned time characteristics result from the source differential equation, with the form and resulting from it the detailed configurations (referred to different fragments of reality) which are given in literature [4-10]. The general differential equation has the following form:

$$
d Z= \pm \frac{\partial Z}{\partial N} d N
$$

where: $d Z$ - total differential of dependent variable; $d N$ - total differential of independent variable; $\partial Z / \partial N$ - partial derivative of dependent variable, referred to independent variable. Signs $( \pm)$ are the algebraic operators which fulfill a determined role. The sign $(+)$ has a formal meaning which just confirms physical sense of a determined dependence. The sign (-) provides such a sense to a determined record [11-15].

The considered variables here are: angular path length $\varphi$ of the pendulum weight, as well as time $t$ of the weight motion. These parameters determine the instantaneous position of the body, considered against its starting/initial interstate position (Fig. 1).

For the considered variables the formula (1) takes the form:

$$
d \varphi= \pm \frac{\partial \varphi}{\partial t} d t
$$

with a dependent variable being the path length $\varphi$, and the independent variable - time $t$. It is worth adding, that the differential equation for a retarded motion will contain the operator $(-)$ , whereas the accelerated motion will be described by equation of this type with the sign $(+)$, which, as can be noticed, does not have to be derived.

Thus for the accelerated motion, taking place between the initial $0-0$ and central $1-1$ positions of the pendulum weight, that is between the following instantaneous static potential fields: unstable $(A S P F)^{*}$ and stable $(S S P F)^{*}$, the equation (2) will assume the following form:

$$
d \varphi=\frac{\partial \varphi}{\partial t} d t
$$




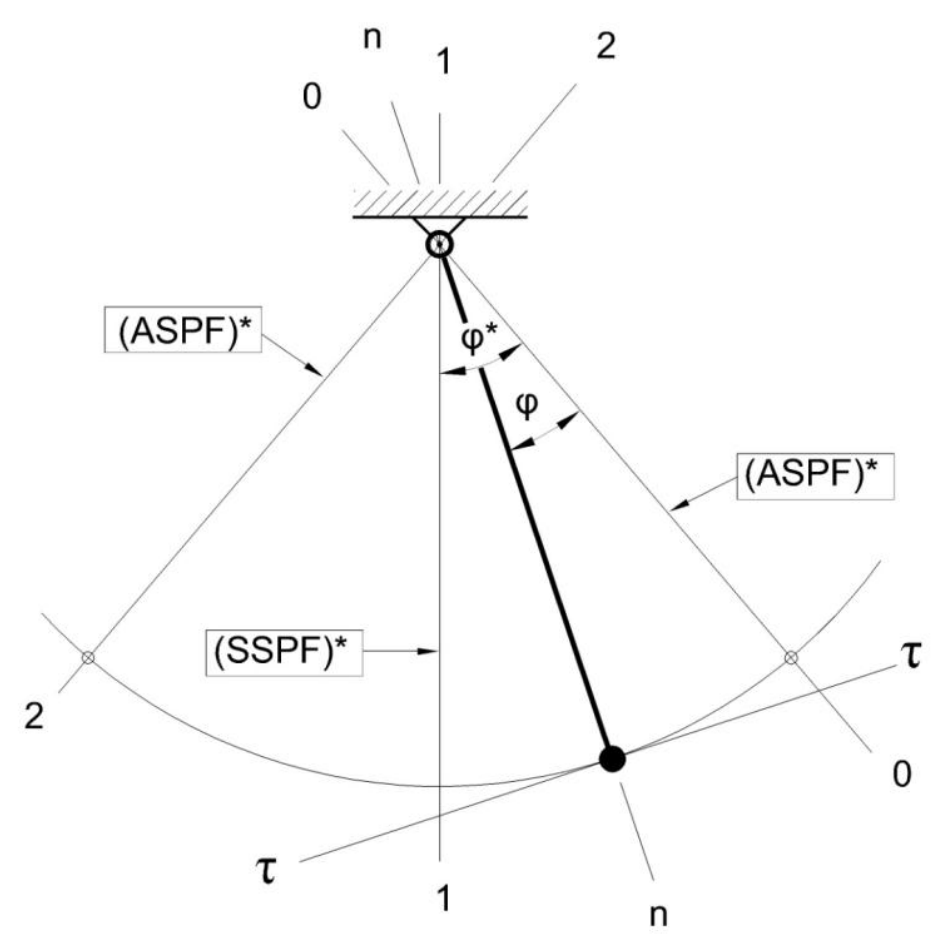

Fig. 1. Interstate position of pendulum weight.

For the considered variables the formula (1) takes the form:

$$
d \varphi= \pm \frac{\partial \varphi}{\partial t} d t
$$

with a dependent variable being the path length $\varphi$, and the independent variable - time $t$. It is worth adding, that the differential equation for a retarded motion will contain the operator $(-)$ , whereas the accelerated motion will be described by equation of this type with the sign $(+)$, which, as can be noticed, does not have to be derived.

Thus for the accelerated motion, taking place between the initial $0-0$ and central $1-1$ positions of the pendulum weight, that is between the following instantaneous static potential fields: unstable $(A S P F)^{*}$ and stable $(S S P F)^{*}$, the equation (2) will assume the following form:

$$
d \varphi=\frac{\partial \varphi}{\partial t} d t
$$


Now two-side integrating of the dependence is needed taking into consideration that the total differential is the state function. That requires further determination of the states which are the limits of integrating: lower and upper.

A scheme of creating the adequate description of dependence $\varphi=f(t)$ is presented in Fig. 2 with all elements of the reasoning process. The curve illustrating that dependence comes out of the initial point of coordinates $t=0, \varphi=0$, and then it takes exponential and progressively rising course. It terminates its course in the point 1 , where the accelerated motion of pendulum weight has its end.

$$
\varphi=\varphi^{*}\left(e^{\frac{t}{\theta}}-1\right)
$$

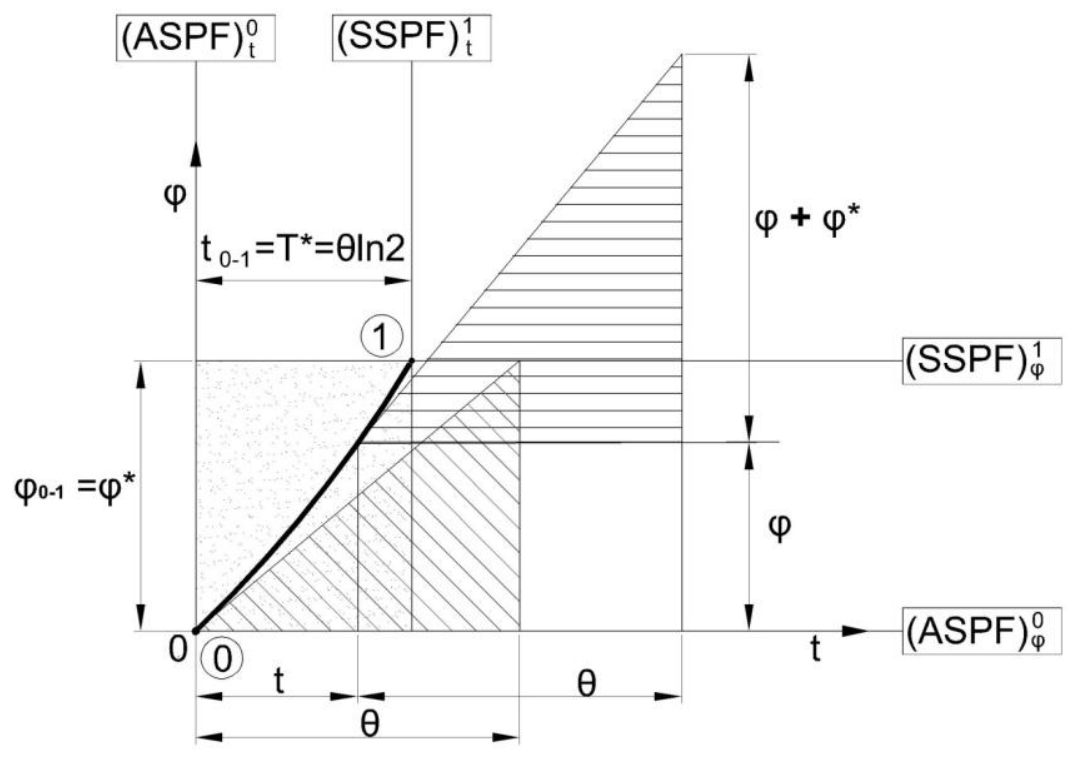

Fig. 2. Indicatrix of dependence of the pendulum weight path length on time of its accelerated motion.

The mentioned limits are potential fields. They were discussed before [5-8] and here treated in more detail. They are referred to both considered physical magnitudes that is the angular path length $\varphi$ and time $t$. There are the following instantaneous fields: angular unstable static potential field $(A S P F)_{\varphi}^{0}$, time unstable static potential field $(A S P F)_{t}^{0}$, angular stable static potential field $(S S P F)_{\varphi}^{1}$, and time stable static potential field $(S S P F)_{t}^{1}$. All these fields are the limits of space-time (dotted area). 
Progressively increasing the exponential curve is the result of moving the rectangular triangle with the horizontal leg being constant and equal time constant $\Theta$, whereas the vertical leg varies respectively, being the sum of coordinate $\varphi$ and the length $\varphi^{*}$ of spacetime.

Time constant $\Theta$ is the time corresponding with the position of crossing point of tangent to the curve of path length (in an initial point) with the line of neighbouring position of angular potential field, that is $(S S P F)_{\varphi}^{1}$. The time constant $\Theta$ is the time coordinate, abscissa of the mentioned point. That time constant may be interpreted in other way, namely: it is the transition time of pendulum weight, the transition with initial velocity on the neighbouring potential field.

It results from the definition of the initial angular velocity, that is the quotient

$$
\omega_{0}=\frac{\varphi^{*}}{\Theta}
$$

where, as noticed, the symbol $\varphi^{*}$ determines the length of space-time (distance between the neighbouring potential fields, considered in the direction of angular path length $\varphi$ ). Therefore

$$
\Theta=\frac{\varphi^{*}}{\omega_{0}}
$$

Now, by integrating the equation (3), the limits of integrals of the total differentials are marked, then

$$
\int_{\varphi}^{2 \varphi+\varphi^{*}} d \varphi=\frac{\partial \varphi}{\partial t} \int_{t}^{t+\Theta} d t
$$

and further

$$
\varphi+\varphi^{*}=\frac{d \varphi}{d t} \Theta
$$

or

$$
\frac{d \varphi}{\varphi+\varphi^{*}}=\frac{1}{\Theta} d t
$$

As can be noticed, the partial derivative has been substituted by the quotient of common/total differentials. It could be done that way because the total differentials have been firmly determined - by introducing limits of their integrals.

Further on, by integrating both sides of equation (8), one obtains the following result 


$$
\ln \left(\varphi+\varphi^{*}\right)=\frac{1}{\Theta} t+C^{*}
$$

that is

$$
\varphi+\varphi^{*}=e^{\frac{t}{\Theta}+C^{*}}=e^{C^{*}} \cdot e^{\frac{t}{\Theta}}=C e^{\frac{t}{\Theta}}
$$

By regarding that for $t=0$ the magnitude $\varphi=0$, one obtains

$$
C=\varphi^{*}
$$

and after substituting (11) to (10)

$$
\varphi=\varphi^{*}\left(e^{\frac{t}{\Theta}}-1\right)
$$

Now one may determine the second coordinate of point 1 , that is $t_{0-1}$. It is obtained by introducing the parameter $\varphi^{*}$ and the mentioned coordinate to equation (12). Therefore

$$
t_{0-1}=\Theta \ln 2
$$

and

$$
\Theta=\frac{t_{0-1}}{\ln 2}
$$

It is worth adding, that $t_{0-1}$ corresponds with a quarter-period $T^{*}$ of pendulum vibrations. Thus

$$
t_{0-1}=T^{*}=\Theta \ln 2
$$

and the whole period of vibrations $T$ contains four those quarter-periods $T^{*}$, that is

$$
T=4 T^{*}
$$

For a retarded pendulum motion, taking place in the time corresponding with the second quarter-period $T^{*}$, that is occurring between the fields $1-1$ and $2-2$ (see Fig. 2), the equation (2) assumes the following form

$$
d \varphi=-\frac{\partial \varphi}{\partial t} d t
$$


Now integrating of the dependence on both sides is to be performed, taking into consideration that the total differential is the state function. That again requires determination of states that is limits of integrating: bottom and top.

The scheme of creation of adequate description of the dependence $\varphi=f(t)$ is presented in Fig. 3 - revealing all elements of that reasoning. The curve illustrating that dependence comes out of the initial point of coordinates $t=0, \varphi=0$, and further it has exponential and degressively rising course. It terminates its real course in the point 2 , where the retarded motion of the pendulum weight has its end.

$$
\varphi=2 \varphi^{*}\left(1-e^{-\frac{t}{\theta}}\right)
$$

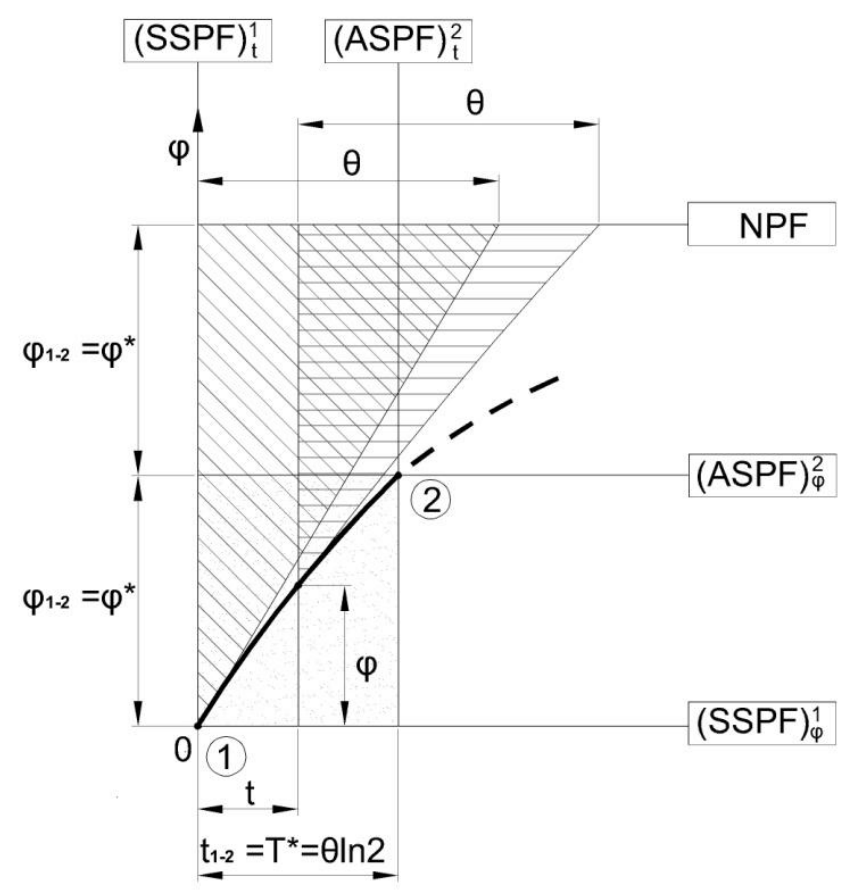

Fig. 3. Indicatrix of dependence of the pendulum weight path length on time of its retarded motion.

The mentioned limits, concerning the studied reality, are the potential fields; they were discussed before. Further on, an additional field is to be introduced, namely the nominal potential field $N P F$. Its essence will be clarified next.

The curve of the path length, contained between the points $1-2$, is the envelope of rectangular triangles, moving by its horizontal leg onto the mentioned nominal potential field; here the horizontal leg is invariable and equaled to the time constant $\Theta$, and the vertical leg varies respectively, decreasing during transition of the triangle in the time direction. That 
nominal field is situated symmetrically against the level of end of the described reality, in the distance from it equal to the length of real space-time in the direction of path length, that is $\varphi_{1-2}=\varphi^{*}$.

Therefore, over the real/proper space-time (dotted area), there is an improper spacetime situated, where the curve (dashed line) tends toward the asymptote, being the mentioned potential field. That is an auxiliary design needed to describe the real curve, reflecting the dependence of $\varphi$ upon $t$.

Now one may begin integrating the equation (17). By integrating that equation, the limits of integrals of the total differentials should be determined. That means

$$
\int_{\varphi}^{2 \varphi^{*}} d \varphi=-\frac{\partial \varphi}{\partial t} \int_{t}^{t+\Theta} d t
$$

and further

$$
2 \varphi^{*}-\varphi=-\frac{d \varphi}{d t} \Theta
$$

or

$$
\frac{d \varphi}{2 \varphi^{*}-\varphi}=-\frac{1}{\Theta} d t
$$

One may notice that here the partial derivative has been substituted by the quotient of total differentials as the total differentials have been clearly determined by introducing the limits of their integrals.

Further on, by integrating both sides of equation (20), one obtains the following result

$$
\ln \left(2 \varphi^{*}-\varphi\right)=-\frac{1}{\Theta} t+C^{*}
$$

that is

$$
2 \varphi^{*}-\varphi=e^{-\frac{t}{\Theta}+C^{*}}=e^{C^{*}} \cdot e^{-\frac{t}{\Theta}}=C e^{-\frac{t}{\Theta}}
$$

By regarding that for $t=0, \varphi=0$

$$
C=2 \varphi^{*}
$$

and after substituting (23) to (22)

$$
\varphi=2 \varphi^{*}\left(1-e^{-\frac{t}{\Theta}}\right)
$$


One should explain that the description is related to the next, new phenomenon, that is the phenomenon of retarded variable motion; this is why the initial conditions were $(t=0, \varphi=0)$.

Now one may determine the second coordinate of point 2 , that is $t_{1-2}$. It is obtained by introducing the parameter $\varphi^{*}$ and the mentioned time coordinate to equation (24). Therefore

$$
t_{1-2}=\Theta \ln 2
$$

and

$$
\Theta=\frac{t_{1-2}}{\ln 2}
$$

Thus the equation (24) is determined mathematically for $t \in\langle 0,+\infty)$, and physically for $t \in\left\langle 0, t_{1-2}\right\rangle$. Of course, only that second interval of time variability will be taken into account, because that adequate, real description of the reality is of importance.

Because $t_{0-1}=t_{1-2}$, the time constant $\Theta$ determined by formulae (14) and (26) is similar and equal

$$
\Theta=\frac{T^{*}}{\ln 2}
$$

That means it is directly proportional to the quarter-period $T^{*}$ of pendulum vibrations, with the coefficient of proportionality being the inverse of $\ln 2$, that is $1: \ln 2$.

\section{KINETIC DERIVATIVES OF THE PENDULUM WEIGHT MAGNITUDE}

It is worth explaining here the difference between kinetics and kinematics. Kinetics, as it is explained in the literature [4,8], characterizes the body motion without regarding its mass. It is about the motion widely understood, both variable, and uniform motion. Kinematics takes into account the body mass but refers only to the uniform motion. Dynamics, as it results is connected with the variable motion of the body of a determined mass.

The kinetics problem of the simple mathematical pendulum is very important; it presents the starting point to more general considerations connected with the dynamics and energy of the device. It is worth describing more deeply all these phenomena - not only for the cognitive reasons, but also for utilitarian and practical ones.

The first derivative magnitude is the velocity, here the angular velocity $\omega$ of pendulum weight. It is the first derivative of angular path length $\varphi$ against time $t$. Therefore, for the accelerated motion, with the first kinematic magnitude (path length) described by the formula (12), the angular velocity $\omega$ is expressed by dependence 


$$
\omega=\frac{d \varphi}{d t}=\frac{d\left[\varphi^{*}\left(e^{\frac{t}{\Theta}}-1\right)\right]}{d t}=\frac{\varphi^{*}}{\Theta} e^{\frac{t}{\Theta}}=\omega_{0} e^{\frac{t}{\Theta}}
$$

where $\omega_{0}$ is the initial angular velocity. It has been described above, by formula (4). The formula (28) defines that velocity by analogy.

Next a kinetic magnitude, that is the angular acceleration $\varepsilon$, being the first derivative of the angular velocity $\omega$, and the second derivative of the path length $\varphi$, is expressed for this motion (accelerated motion) by the following dependence

$$
\varepsilon=\frac{d \omega}{d t}=\frac{d^{2} \varphi}{d t^{2}}=\frac{\omega_{0}}{\Theta} e^{\frac{t}{\Theta}}=\frac{\varphi^{*}}{\Theta^{2}} e^{\frac{t}{\Theta}}=\varepsilon_{0} e^{\frac{t}{\Theta}}
$$

For a complete set of moments, that is for the terminal values of the time of accelerated motion, that is $t=0$ and $t=T^{*}=\Theta \ln 2$, the angular velocity $\omega$ equals respectively

$$
\omega=\omega_{0}
$$

and

$$
\omega=\omega_{1}=2 \omega_{0}
$$

and acceleration

$$
\varepsilon=\varepsilon_{0}
$$

and

$$
\varepsilon=\varepsilon_{1}=2 \varepsilon_{0}
$$

For a retarded motion, for which the path length is described by the formula (24), the particular derivative kinetic magnitudes are expressed by the formulae

$$
\begin{gathered}
\omega=\frac{d \varphi}{d t}=\frac{d\left[2 \varphi^{*}\left(1-e^{-\frac{t}{\Theta}}\right)\right]}{d t}=\frac{2 \varphi^{*}}{\Theta} e^{-\frac{t}{\Theta}}=2 \omega_{0} e^{-\frac{t}{\Theta}}=\omega_{1} e^{-\frac{t}{\Theta}} \\
\varepsilon=\frac{d \omega}{d t}=\frac{d^{2} \varphi}{d t^{2}}=\frac{2 \omega_{1}}{\Theta} e^{-\frac{t}{\Theta}}=\frac{2 \varphi^{*}}{\Theta^{2}} e^{-\frac{t}{\Theta}}=2 \varepsilon_{0} e^{-\frac{t}{\Theta}}=\varepsilon_{1} e^{-\frac{t}{\Theta}}
\end{gathered}
$$

Corresponding with the terminal values of time period of a retarded motion, that is for $t=0$ and $t=T^{*}=\Theta \ln 2$, the angular velocity $\omega$ equals, respectively 


$$
\omega=\omega_{1}
$$

and

$$
\omega=\omega_{2}=\frac{\omega_{1}}{2}=\omega_{0}
$$

and acceleration

$$
\varepsilon=\varepsilon_{1}
$$

and

$$
\varepsilon=\varepsilon_{2}=\frac{\varepsilon_{1}}{2}=\varepsilon_{0}
$$

All these magnitudes may be naturally connected with the length $l$ of pendulum, by obtaining them for motion in the direction tangent peripheral to the weight track. Thus the equivalents of formulae (28-39) will be the following formulae:

$$
\begin{gathered}
v_{\tau}=\omega \cdot l=\frac{\varphi^{*}}{\Theta} l e^{\frac{t}{\Theta}}=\omega_{0} \cdot l e^{\frac{t}{\Theta}} \\
a_{\tau}=\frac{\omega_{0}}{\Theta} l e^{\frac{t}{\Theta}}=\frac{\varphi^{*}}{\Theta^{2}} l e^{\frac{t}{\Theta}}=\varepsilon_{0} \cdot l e^{\frac{t}{\Theta}} \\
v_{\tau}=v_{\tau}^{0}=\omega_{0} \cdot l e^{\frac{t}{\Theta}} \\
v_{\tau}=v_{\tau}^{1}=2 \omega_{0} \cdot l e^{\frac{t}{\Theta}} \\
a_{\tau}=a_{\tau}^{0}=\varepsilon_{0} \cdot l \\
a_{\tau}=a_{\tau}^{1}=\varepsilon_{1} \cdot l=2 \varepsilon_{0} \cdot l \\
v_{\tau}=\frac{2 \varphi^{*}}{\Theta} l e^{-\frac{t}{\Theta}}=2 \omega_{0} \cdot l e^{-\frac{t}{\Theta}}=\omega_{1} \cdot l e^{-\frac{t}{\Theta}} \\
a_{\tau}=\frac{2 \omega_{0}}{\Theta} l e^{-\frac{t}{\Theta}}=\frac{2 \varphi^{*}}{\Theta{ }^{2}} l e^{-\frac{t}{\Theta}}=2 \varepsilon_{0} \cdot l e^{-\frac{t}{\Theta}}=\varepsilon_{1} \cdot l e^{-\frac{t}{\Theta}} \\
v_{\tau}=v_{\tau}^{1}=\omega_{1} \cdot l
\end{gathered}
$$




$$
\begin{gathered}
v_{\tau}=v_{\tau}^{2}=\omega_{2} \cdot l=\frac{\omega_{1}}{2} l=\omega_{0} \cdot l \\
a_{\tau}=a_{\tau}^{1}=\varepsilon_{1} \cdot l \\
a_{\tau}=a_{\tau}^{2}=\varepsilon_{2} \cdot l=\frac{\varepsilon_{1}}{2} l=\varepsilon_{0} \cdot l
\end{gathered}
$$

In Fig. 4a, a graphical interpretation of the velocity dependence $v_{\tau}$ of the pendulum weight on time, for two first quarter-periods, is presented. Fig. $4 \mathbf{b}$ illustrates the course of dependence of acceleration $a_{\tau}$ for the same quarter-periods. That cycle of changes (both of the velocity and acceleration) will be repeated without changing its configuration.
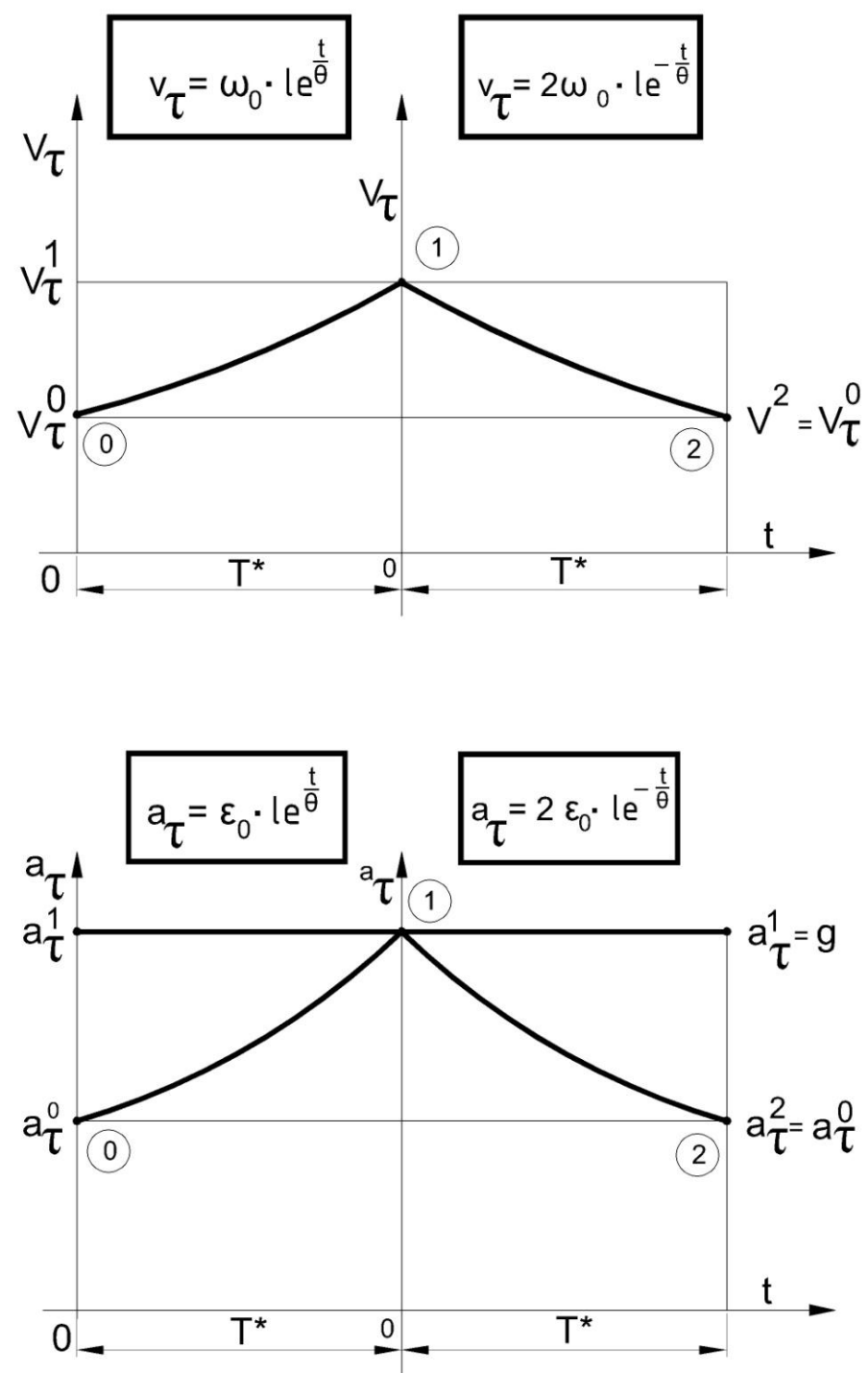

Fig. 4. Graphical interpretation of dependence of the velocity (a) and acceleration (b) of pendulum weight on time for the first two quarter-periods. 
It is worth noticing that the acceleration $a_{\tau}$ in the central point (Fig. 4b), that is $a_{\tau}^{1}$, is equal to the terrestrial acceleration $g$, that is $a_{\tau}^{1}=g$. That equality may be used to derive the formula on the vibration period $T$ of pendulum. Considering the formula (45), one obtains

$$
g=a_{\tau}^{1}=2 \varepsilon_{0} \cdot l
$$

and further, by regarding (41), one obtains

$$
g=2 \frac{\varphi^{*}}{\Theta^{2}} l
$$

After substituting the formula on $\Theta$, that is (27), to (53) one obtains

$$
g=2(\ln 2)^{2} \frac{\varphi^{*}}{\left(T^{*}\right)^{2}} l
$$

and then - knowing that $T^{*}=T: 4$, according to formula (16) - one obtains

$$
g=\frac{(\ln 2)^{2}}{8} \cdot \frac{\varphi^{*}}{T^{2}} \cdot l
$$

from which

$$
T=\frac{\ln 2}{2} \sqrt{\frac{\varphi^{*} \cdot l}{2 g}}
$$

Now a normal acceleration $a_{n}$ should be taken into consideration. That acceleration is expressed by the following, known formula of definition

$$
a_{n}=\frac{\left(v_{\tau}\right)^{2}}{l}=\frac{\omega^{2} \cdot l^{2}}{l}=\omega^{2} \cdot l
$$

All three accelerations (tangent $a_{\tau}$, normal $a_{n}$, and terrestrial $g$ ) have been presented graphically in Fig. 5. As can be seen, in the terminal position of the analyzed pendulum between particular accelerations the following relations occur, $a_{\tau}^{0}\left\langle g\left\langle a_{n}^{0}\right.\right.$. In the intermediate position, the same relations $\left(a_{\tau}\left\langle g\left\langle a_{n}\right)\right.\right.$ will take place, whereas in the central position, $\left.a_{n}^{1}\right\rangle a_{\tau}^{1}=g$. 


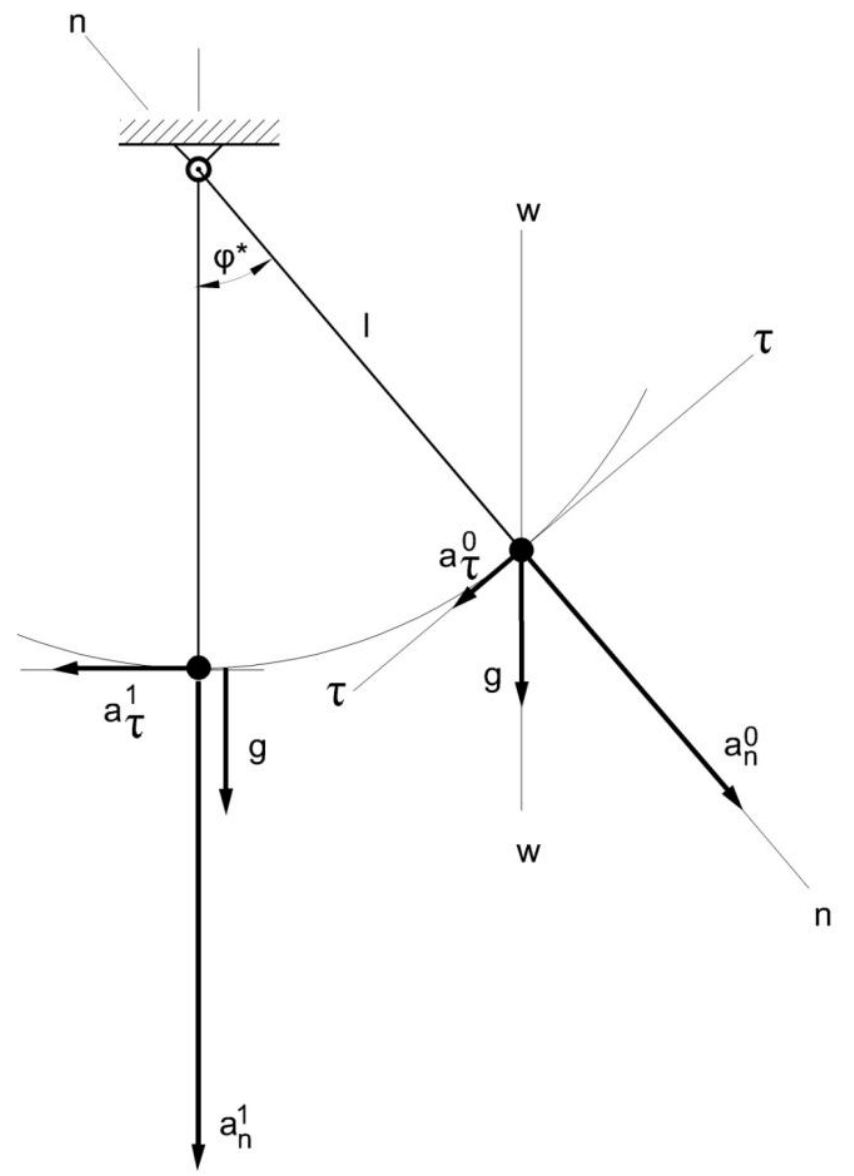

Fig. 5. Accelerations (static $a_{\tau}$, normal $a_{n}$, and terrestrial $g$ ) in the extreme and central position of pendulum.

\section{CONCLUSIONS}

Novel theory of a simple mathematical pendulum varies considerably from the existent theory of motion of that kind of device. The new adequate theory, in opposition to the existing one, describes a real pendulum motion; it is the motion in vertical plane. In that theory the quantum non-continuous character of the variable motion of pendulum weight is clearly indicated. Very carefully in detail are described in the theory the terminal turning points of the weight path with the exact presentation of its specific behaviours. Especially important is the description of its transition from an instantaneous terminal equilibrium to the unbalance, being the condition for beginning of the free variable motion.

The elaborated theory of mathematical pendulum clearly indicates, occurring in the reality, a big differentiation of the weight motion. The variable motion reveals different characteristics between the neighbouring space-times in consecutive quarter-periods. At the beginning, from the moment of pendulum setting in motion (setting it in a free motion) the accelerated free variable motion takes place, then - a retarded motion of that type, and further again the accelerated motion, etc. Different descriptions of the pendulum weight path lengths 
correspond with them along with the descriptions of derivatives of kinetic magnitudes. It is worth noting that the kinetics has an open character; one may determine next kinetic magnitudes, such as impulse and further, not named nor used magnitudes as yet.

It is interesting that one of the recent other works considers "rethinking Newton's Principia" in Galilean space-time [16]. One would say: at last! Though it refers to the universe, the necessity to "rethink" many existent theories is of importance. This work was focused mainly onto the mathematical pendulum kinetics, with the dynamics considered in peculiar points only, not presenting analytically the characteristics of particular components of forces. That was resulting from the need/necessity to explain the pendulum behaviours just in these particular points on the path of its variable motion. The energy and dynamics problems have not been considered due to the frameworks of the work. They are to be presented in the following elaborations.

\section{References}

[1] Zdzisław Pluta, Tadeusz Hryniewicz, International Letters of Chemistry, Physics and Astronomy 9(2) (2013) 136-145 (Part 1).

[2] Zdzisław Pluta, Tadeusz Hryniewicz, International Letters of Chemistry, Physics and Astronomy 8(2) (2013) 113-122.

[3] Zdzisław Pluta, Tadeusz Hryniewicz, International Letters of Chemistry, Physics and Astronomy 8(3) (2013) 195-204.

[4] Zdzisław Pluta, Tadeusz Hryniewicz, Int. J. Adv. Manuf. Technol. 62(5) (2012) 529-542; DOI: $10.1007 / \mathrm{s} .00170-011-3813-5$ (14 pages).

[5] Zdzisław Pluta, Tadeusz Hryniewicz, International Letters of Chemistry, Physics and Astronomy 2 (2012) 28-34.

[5] Zdzisław Pluta, Tadeusz Hryniewicz, International Letters of Chemistry, Physics and Astronomy 3 (2012) 1-10.

[6] Zdzisław Pluta, Tadeusz Hryniewicz, International Letters of Chemistry, Physics and Astronomy 3 (2012) 11-23.

[7] Zdzisław Pluta, Tadeusz Hryniewicz, International Letters of Chemistry, Physics and Astronomy 4 (2012) 1-7.

[8] Zdzisław Pluta, Tadeusz Hryniewicz, Int. J. Adv. Manuf. Technol. 51 (2010) 35-43; DOI: 10.1007/s.00170-010-2595-5 (9pages).

[9] Zdzisław Pluta, Tadeusz Hryniewicz, Tribology Transactions 55(2) (2012) 230-236; http: 101080/10402004.2011.647385

[10] Zdzisław Pluta, Tadeusz Hryniewicz, International Letters of Chemistry, Physics and Astronomy 4 (2012) 8-16.

[11] Zdzisław Pluta, Tadeusz Hryniewicz, International Letters of Chemistry, Physics and Astronomy 5 (2012) 35-45.

[12] Zdzisław Pluta, Tadeusz Hryniewicz, International Letters of Chemistry, Physics and Astronomy 4 (2013) 56-72. 
[13] Zdzisław Pluta, Tadeusz Hryniewicz, International Letters of Chemistry, Physics and Astronomy 6 (2013) 116-136.

[14] Zdzisław Pluta, Tadeusz Hryniewicz, International Letters of Chemistry, Physics and Astronomy 7(2) (2013) 85-101.

[15] Simon Saunders, Philosophy of Science 80(1) (2013) 22-48. 